\title{
Epidermal stem cells ride the circadian wave
}

Vivek Kumar $^{1,2}$, Bogi Andersen ${ }^{3^{*}}$ and Joseph S Takahashi ${ }^{1,2^{*}}$

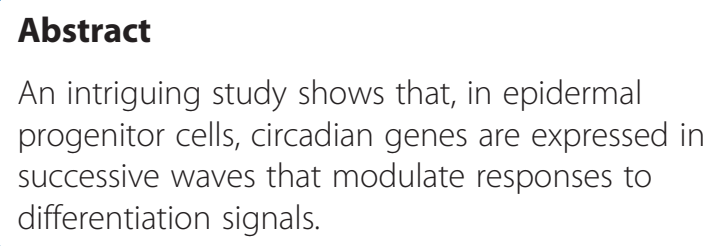

An intriguing study shows that, in epidermal progenitor cells, circadian genes are expressed in successive waves that modulate responses to differentiation signals.

\section{Molecular mechanisms of the circadian clock}

Circadian rhythms coordinate the physiology of an organism with the light-dark environmental variation caused by the rotation of the earth. Most organisms have some form of circadian regulation, making it one of the most ubiquitous regulatory pathways discovered to date. The core circadian components in mammals include an autoregulatory feedback loop in which the positive factors Clock and Bmal1 (Arntl) dimerize to transactivate expression of negative Per and Cry factors. A secondary loop includes transactivation of genes encoding Ror factors and Rev-Erb that, respectively, activate and repress target genes, including Bmall. In mammals, the circadian clock is cell-autonomous - each individual cell has a self-sustaining clock. However, only cells in the eye can sense light information, which is conveyed to the suprachiasmatic nucleus $(\mathrm{SCN})$ - the master pacemaker in the hypothalamus - through a pathway known as the retinohypothalamic tract. The $\mathrm{SCN}$ in turn coordinates clocks in peripheral tissues in order to regulate physiology and behavior [1]. Although the functions and mechanisms of central core clock genes have been studied extensively, their roles in peripheral tissues remain largely unknown. In a recent study, however, Janich et al. begin to fill this gap, as they describe an unexpected function of clock genes in the regulation of human skin differentiation [2].

\footnotetext{
* Correspondence: bogi@uci.edu; joseph.takahashi@utsouthwestern.edu ${ }^{3}$ Department of Biological Chemistry, University of California Irvine, Irvine, California, USA

${ }^{1}$ Department of Neuroscience, University of Texas Southwestern Medical Center, Dallas, TX, USA

Full list of author information is available at the end of the article
}

\section{The circadian clock and the skin}

The skin takes the brunt of damage from the harmful radiation emitted by the sun and is constantly exposed to toxins and abrasive injuries. The outmost layer of the skin, the epidermis, comprises a stratified squamous epithelium that is maintained by proliferating stem and progenitor cells residing in the basal cell layer. These progenitors exit the cell cycle as they enter the suprabasal compartment, where they undergo a series of differentiation steps, ultimately forming the dead cornified layer at the surface of the skin. While the role of the circadian clock in regulation of diurnal changes of physiology through modulation of gene expression in metabolically active organs, such as the liver, fat and muscle, is well known, less is known for epithelial tissues, such as the skin, which primarily perform protective functions. Over 50 years ago, it was observed that cell proliferation in the skin occurs in a circadian manner [3]. However, only recently, by utilizing mice harboring mutations in core clock genes, has the functional significance of this regulation become clear [4-7]. Furthermore, mouse skin is more sensitive at night than during the day to DNA damage and tumorigenesis from ultraviolet $B$ (UVB) radiation; the greater sensitivity to UVB radiation correlates with there being a higher proportion of progenitors in $S$ phase and less efficient excision repair of DNA at night $[4,8]$. The evolutionary advantage underlying the circadian clock regulation of cell proliferation in the epidermis remains unclear, but it could relate to improved function of progenitor and stem cells when DNA replication is temporally separated from the maximum generation of reactive oxygen species from oxidative phosphorylation [4].

\section{Multiple waves of gene expression in synchronized normal human epidermal keratinocyte cells}

To investigate the role of the circadian clock in epidermal cells, Janich et al. utilized an in vitro model in which normal human epidermal keratinocytes (NHEKs), cultured under conditions of low $\mathrm{Ca}^{2+}$, resemble progenitor cells of the basal cell layer. NHEKs were induced to differentiate 
by increasing the concentration of $\mathrm{Ca}^{2+}$, during which they executed a gene expression program bearing similarity to that of suprabasally located keratinocytes. When synchronizing the clock of cultured NHEKs, the authors discovered unexpected complexity in circadian clock gene expression in these cells [2]. Core circadian genes fell into five phases of peak mRNA levels. These phases, termed A to E, corresponded with peak expression of the genes for the nuclear receptors NR1D1 and NR1D2 (phase A), the period circadian proteins PER1 and PER3 (phase B), the period circadian protein PER2 and the cryptochrome CRY2 (phase $\mathrm{C}$ ), the cryptochrome CRY1 (phase D) and the aryl hydrocarbon receptor BMAL1 (ARNTL) (phase E) (Table 1).

Whole-genome expression analysis showed between 720 (peak A, Table 1) and 1,667 (peak C, Table 1) active genes in each of these five phases under the two conditions - undifferentiated and differentiating - with little overlap of genes in each phase between undifferentiated and differentiating keratinocytes. Each phase is enriched for a unique set of gene ontology (GO) categories. Analysis of the enriched GO categories indicates that peaks A to $\mathrm{C}$, corresponding to late-night to early-morning hours, encompass pathways involved in keratinocyte differentiation, whereas peaks $\mathrm{D}$ and $\mathrm{E}$, corresponding to afternoon to evening hours, are pathways involved in DNA replication, UV protection and cell division. Thus, the phases of circadian gene oscillation correlate with different biological and physiological functions, causing differentiation versus DNA replication and repair to occur at distinct circadian times. The work also suggests that the sensitivity of keratinocytes to certain signaling pathways is circadian time dependent.

\section{Circadian time dictates sensitivity to signaling pathways}

Janich et al. also investigated in detail two major differentiation pathways in keratinocytes, involving $\mathrm{Ca}^{2+}$ and transforming growth factor beta (TGF $\beta$ ). The genes associated with these two categories are enriched in peaks $\mathrm{B}$ and $\mathrm{C}$, corresponding to differentiation phases of late night to early morning. The authors found that the gene targets of $\mathrm{Ca}^{2+}$ and TGF $\beta$ signaling are most responsive at the time of peaks B or C in synchronized NHEK cells. This difference in sensitivity implies that circadian conditioning of these signal transduction pathways occurs such that the same treatment elicits a greater response at certain times. Perturbation of circadian rhythms by overexpressing the genes encoding PER1 or PER2 or by knocking down $C R Y 1$ or $C R Y 2$ led to premature differentiation in in vitro assays and colonization defects in orthotopic transplantation studies in mice.

Why there should be an evolutionary advantage of initiating the epidermal differentiation program at specific times during the day remains a mystery, especially considering that the transit time of a progenitor cell from the basal cell layer to the surface of the human skin is very long - between 1 and 2 months. The end-product of differentiation - the cornified envelope - persists for a long time. Furthermore, despite abnormalities in cell proliferation dynamics, none of the core clock mutants in mice exhibits obvious barrier or differentiation defects of the interfollicular epidermis. There is some evidence of an age-dependent decrease in skin function in Bmal1 (Arntl) knockout mice [9]. One possibility is that circadian variation in the ability to induce differentiation is secondary to the effect of the circadian clock on cell proliferation, given that only post-mitotic cells normally enter the differentiation program.

\section{Moving from in vitro to in vivo human studies}

The report by Janich et al. is an exciting contribution to the growing field of circadian clock studies in epithelial tissues. However, the induction by $\mathrm{Ca}^{2+}$ of differentiation in NHEK cultures is by no means a perfect model of

Table 1 The peaks of circadian gene oscillation and their correlation with different biological and physiological functions

\begin{tabular}{|c|c|c|c|c|c|c|c|}
\hline \multirow[t]{2}{*}{ Peak } & \multirow{2}{*}{$\begin{array}{l}\text { Clock } \\
\text { genes }\end{array}$} & \multirow{2}{*}{$\begin{array}{l}\text { Peak time } \\
\text { (hours after } \\
\text { synchronization) }\end{array}$} & \multicolumn{3}{|c|}{ Number of genes } & \multirow[t]{2}{*}{ Pathways } & \multirow[t]{2}{*}{ Cellular stat } \\
\hline & & & Differentiated & $\begin{array}{l}\text { Stem } \\
\text { cell }\end{array}$ & Both & & \\
\hline $\bar{A}$ & $\begin{array}{l}\text { NR1D1, } \\
\text { NR1D2 }\end{array}$ & 16 & 327 & 374 & 19 & $\begin{array}{l}\text { Protein localization, transcriptional regulation, cytoskeleton, cAMP } \\
\text { metabolism, collagen metabolism }\end{array}$ & Differentiation \\
\hline B & $\begin{array}{l}\text { PER1, } \\
\text { PER3 }\end{array}$ & 20 & 566 & 454 & 28 & $\begin{array}{l}\mathrm{Ca}^{2+} \text { homeostasis, cholesterol metabolism, RNA modification, } \\
\text { amino acid metabolism, vitamin D response, ribosome biogenesis }\end{array}$ & \\
\hline$C$ & $\begin{array}{l}\text { PER2, } \\
\text { CRY2 }\end{array}$ & 22 & 899 & 695 & 73 & $\begin{array}{l}\mathrm{Ca}^{2+} \text { homeostasis, glucocorticoid response, glucose and lipid } \\
\text { metabolism, ribosome biogenesis, cell cycle }\end{array}$ & \\
\hline $\mathrm{D}$ & CRY1 & 26 & 494 & 575 & 23 & $\begin{array}{l}\text { Nuclear lumen organization, DNA damage response, repair, } \\
\text { ribosome biogenesis, mitochondrial morphogenesis, cell cycle } \\
\text { and DNA replication, splicing regulation, pigmentation, ATP } \\
\text { metabolism }\end{array}$ & $\begin{array}{l}\text { DNA } \\
\text { replication, } \\
\text { repair }\end{array}$ \\
\hline E & BMAL1 & 34 & 832 & 415 & 23 & $\begin{array}{l}\text { Endosome membrane, RNA localization, chromatin remodeling, } \\
\text { DNA metabolism, stress response, cell division, mitotic phase }\end{array}$ & \\
\hline
\end{tabular}


human epidermal differentiation. Furthermore, the synchronization method used in this study - serum shock - can directly synchronize the cell cycle, which is often approximately 24 hours in cultured cells, confounding gene expression changes primarily associated with the cell cycle with those primarily linked to the circadian clock. A better model for circadian control of human epidermal differentiation is primary intact epidermis collected at different times of the day, as utilized in a study by Spörl et al. [10], as well as distinct cell populations from the intact epidermis.

In today's 24-hour society, where sleep deprivation and circadian disruption are the norm, determining the exact role of the circadian rhythm in skin physiology and disease is an important topic for human health. Current work in the field suggests that the circadian clock is an important regulator of epidermal cell proliferation and skin cancer, as well as skin aging, and is a major contributor to chronic wounds in the elderly [8-10]. Thus, we see here another example of the role of circadian clocks in adaptive cellular physiology.

\section{Abbreviations}

GO: Gene ontology; NHEK: Normal human epidermal keratinocyte; SCN: Suprachiasmatic nucleus; TGF $\beta$ : Transforming growth factor beta; UVB: Ultraviolet B.

\section{Competing interests}

The authors declare that they have no competing interests.

\section{Author details}

'Department of Neuroscience, University of Texas Southwestern Medical Center, Dallas, TX, USA. ${ }^{2}$ Howard Hughes Medical Institute, University of Texas Southwestern Medical Center, Dallas, TX, USA. ${ }^{3}$ Department of Biological Chemistry, University of California Irvine, Irvine, California, USA.

Published: 29 November 2013

\section{References}

1. Lowrey PL, Takahashi JS: Genetics of circadian rhythms in mammalian model organisms. Adv Genet 2011, 74:175-230.

2. Janich P, Toufighi K, Solanas G, Luis NM, Minkwitz S, Serrano L, Lehner B, Benitah SA: Human epidermal stem cell function is regulated by circadian oscillations. Cell Stem Cell 2013, 13:1-9.

3. Scheving LE: Mitotic activity in the human epidermis. Anat Rec 1959, 135:7-19.

4. Geyfman M, Kumar V, Liu Q, Ruiz R, Gordon W, Espitia F, Cam E, Millar SE, Smyth P, Ihler A, Takahashi JS, Andersen B: Brain and muscle Arnt-like protein-1 (BMAL1) controls circadian cell proliferation and susceptibility to UVB-induced DNA damage in the epidermis. Proc Natl Acad Sci U S A 2012, 109:11758-11763.

5. Janich P, Pascual G, Merlos-Suárez A, Batlle E, Ripperger J, Albrecht U, Cheng HY, Obrietan K, Di Croce L, Benitah SA: The circadian molecular clock creates epidermal stem cell heterogeneity. Nature 2011, 480:209-214.

6. Lin KK, Kumar V, Geyfman M, Chudova D, Ihler AT, Smyth P, Paus R, Takahashi JS, Andersen B: Circadian clock genes contribute to the regulation of hair follicle cycling. PLoS Genet 2009, 5:e1000573.

7. Plikus MV, Vollmers C, de la Cruz D, Chaix A, Ramos R, Panda S, Chuong CM: Local circadian clock gates cell cycle progression of transient amplifying cells during regenerative hair cycling. Proc Natl Acad Sci U S A 2013, 110: E2106-E2115.

8. Gaddameedhi S, Selby CP, Kaufmann WK, Smart RC, Sancar A: Control of skin cancer by the circadian rhythm. Proc Natl Acad Sci U S A 2011, 108:18790-18795.
9. Kondratov RV, Kondratova AA, Gorbacheva VY, Vykhovanets OV, Antoch MP: Early aging and age-related pathologies in mice deficient in BMAL1, the core component of the circadian clock. Genes Dev 2006, 20:1868-1873.

10. Spörl F, Korge S, Jürchott $K$, Wunderskirchner M, Schellenberg K, Heins S, Specht A, Stoll C, Klemz R, Maier B, Wenck H, Schrader A, Kunz D, Blatt T, Kramer A: Krüppel-like factor 9 is a circadian transcription factor in human epidermis that controls proliferation of keratinocytes. Proc Natl Acad Sci U S A 2012, 109:10903-10908.

doi:10.1186/gb4142

Cite this article as: Kumar et al:: Epidermal stem cells ride the circadian wave. Genome Biology 2013 14:140. 